\title{
Akutes Koronarsyndrom
}

\section{Praxisbesonderheit bei der antithrombozytären Therapie}

Der Gemeinsame Bundesausschuss hat Ticagrelor einen Zusatznutzen für die überwiegende Mehrheit der Patienten mit akutem Koronarsyndrom attestiert. Daher gilt die Verordnung des Thrombozytenaggregationshemmers in diesen Fällen als Praxisbesonderheit.

Die Verordnung eines neuen Arzneimittels, dem der Gemeinsame Bundesausschuss (G-BA) einen Zusatznutzen zugesprochen und für das der Spitzenverband der gesetzlichen Krankenkassen (GKV) mit dem Hersteller einen Preis vereinbart hat, gilt als Praxisbesonderheit. Ticagrelor (Brilique ${ }^{T M}$ ) ist das erste Medikament, für das die Preisverhandlungen nach den Vorgaben des Arzneimittelmarktneuordnungsgesetzes (AMNOG) erfolgreich abgeschlossen wurden, und das in diesem Zusammenhang den Status als Praxisbesonderheit erhielt.
Laut G-BA wird für Patienten mit NichtST-Strecken-Hebungsinfarkt (NSTEMI) sowie für Patienten mit instabiler Angina pectoris durch den Einsatz von Ticagrelor im Vergleich zu Clopidogrel ein beträchtlicher Zusatznutzen erreicht. Ein zusätzlicher Nutzen wird auch für Patienten mit ST-StreckenHebungsinfarkt (STEMI) und perkutaner Koronarintervention bescheinigt, die älter als 75 Jahre sind, nach einer individuellen Nutzen-Risiko-Abwägung nicht für eine Therapie mit Prasugrel infrage kommen oder einen ischämischen Schlaganfall oder eine transitorische ischämische Attacke hatten. In den genannten vom G-BA anerkannten Zusatznutzen-Indikationen kann Ticagrelor laut Prof. Evangelos Giannitsis vom Universitätsklinikum Heidelberg verordnet werden, ohne dass diese Verordnungen im Fall einer Richtgrößenprüfung im Budget angerechnet werden.
Wie Giannitsis informierte, stützt sich der Beschluss des G-BA im Wesentlichen auf die Daten, die in der PLATO-Studie gewonnen wurden.,In der internationalen Multicenterstudie wurde gezeigt, dass Ticagrelor bei Patienten mit akutem Koronarsyndrom im Vergleich zu Clopidogrel die absolute Häufigkeit des kombinierten Endpunkts aus kardiovaskulär bedingtem Tod, Myokardinfarkt und Schlaganfall nach zwölf Monaten signifikant senken konnte", sagte Giannitsis (Ticagrelor 9,8\% vs. Clopidogrel 11,7\%; $p<$ $0,001)$. Darüber hinaus sei auch die Myokardinfarktrate im Ticagrelor-Arm signifikant niedriger gewesen, so der Kardiologe (5,8\% vs. $6,0 \% ; p=0,005)$.

(wed)

Satellitensymposium, DGK-Herbsttagung, Hamburg, 12. Oktober 2012 (Veranstalter: AstraZeneca)

\section{Zielindikation Vorhofflimmern}

\section{Zuwachs in der Antikoagulanzien-Familie}

\section{Vitamin-K-Antagonisten haben bekanntlich Konkurrenz bekommen. Eine der wichtigsten Zielindikationen der neuen oralen Antikoagulanzien ist die Schlaganfallprophylaxe bei Patien- ten mit Vorhofflimmern. Das trifft auch für das noch nicht zugelassene Edoxa- ban (Lixiana ${ }^{\oplus}$ ) zu, das derzeit in einer großen Phase-III-Studie weltweit ge- prüft wird.}

Edoxaban ist ein spezifischer und reversibler Faktor-Xa-Hemmer, dessen Herkunftsland der Substanzname verrät: Edo ist der alte Name für Tokyo, Xaban das chemische Kürzel für Faktor-Xa-Hemmer. Diese Neuentwicklung wird nur zu 33\% renal ausgeschieden und hat eine Bioverfügbarkeit von $45 \%$.
Wie bei den anderen neuen oralen Antikoagulanzien entfällt auch bei Edoxaban regelmäßiges Gerinnungsmonitoring - ein großes Plus gegenüber den Vitamin-K-Antagonisten.

Das Studienprogramm hat mit dem Nachweis der Sicherheit und Wirksamkeit bei venösen Thromboembolien begonnen und ist inzwischen mit der Megastudie ENGAGE-AF-TIMI-48 auf die Indikation Schlaganfallprophylaxe bei Vorhofflimmern ausgeweitet worden. ENGAGE-AF steht für ${ }_{\text {"Effec- }}$ tive aNticoaGulation with Faktor XA Next Generation in Atrial Fibrillation". Es ist bisher die größte Doppelblindstudie mit Antikoagulanzien bei Vorhofflimmern: 21107 Patienten sind in 1400 Zentren weltweit rekrutiert worden, eine Phase-III-Studie, in der zwei unterschiedliche Dosierungen von Edo- xaban (einmal täglich $30 \mathrm{mg}$ oder $60 \mathrm{mg}$ ) mit Warfarin (INR zwischen 2 und 3) verglichen werden. Primärer Endpunkt ist die Rate der Schlaganfälle oder systemischen Embolien nach einer Studiendauer von mindestens 24 Monaten. Zu den Einschlusskriterien gehören ein dokumentiertes Vorhofflimmern, das nicht länger als zwölf Monate anhält sowie ein CHADS2-Score von mindestens 2.

Die Ergebnisse werden im Frühjahr 2013 erwartet. Erst dann kann beurteilt werden, wie Edoxaban im Vergleich zu Warfarin und anderen neuen oralen Antikoagulanzien abschneidet.

(JA) II

II Symposium bei der DGK-Herbsttagung, Hamburg, 11. Oktober 2012 (Veranstalter: Daiichi-Sankyo) 